\title{
ESTRATÉGIAS DE CAPTAÇÃO DE DOADORES DE SANGUE: UMA REVISÃO INTEGRATIVA DA LITERATURA ${ }^{1}$
}

\author{
Rosane Suely May Rodrigues ${ }^{2}$, Kenya Schmidt Reibnitz ${ }^{3}$
}

\footnotetext{
${ }^{1}$ Parte integrante da tese - Avaliação de impacto do projeto escola na captação de doadores do Centro de Hematologia e Hemoterapia de Santa Catarina de Florianópolis, apresentada ao Programa de Pós-Graduação em Enfermagem (PEN) da Universidade Federal de Santa Catarina (UFSC), 2011.

2 Doutoranda em Enfermagem do PEN/UFSC. Assistente Social do Centro de Hematologia e Hemoterapia de Santa Catarina. Santa Catarina, Brasil. E-mail: rosanesuely@gmail.com

${ }^{3}$ Doutora em Enfermagem. Docente do Departamento de Enfermagem e do PEN/UFSC. Santa Catarina, Brasil. E-mail: kenya@ ccs.ufsc.br
}

RESUMO: Este estudo trata de uma revisão integrativa da literatura, com o objetivo de conhecer as estratégias de captação de doadores de sangue utilizadas pelos hemocentros. O levantamento bibliográfico abrangeu as publicações nacionais e internacionais de 2000 a 2009, sendo identificados 39 textos que compuseram a amostra do estudo. Os resultados identificaram a escassez de publicação sobre o tema, sendo que os Estados Unidos da América, Brasil, Canadá e Reino Unido foram os países que mais publicaram. As estratégias encontradas para captação de doadores foram agrupadas como: acolhimento; e campanhas e estratégias educativas. Observamos a necessidade de maior socialização das estratégias de captação de doadores de sangue utilizadas pelos hemocentros, a fim de possibilitar a troca de experiências entre os serviços e, dessa forma, contribuir para o aumento da captação de doadores de sangue, na busca de novos doadores e de sua fidelização.

DESCRITORES: Doadores de sangue. Marketing social. Bancos de sangue. Serviço de hemoterapia.

\section{STRATEGIES FOR ATTRACTING BLOOD DONORS: AN INTEGRATIVE LITERATURE REVIEW}

\begin{abstract}
The intention of this integrative literature review is to better know the strategies for attracting blood donors used by blood banks (hemocenters) in Brazil. The bibliographic survey covered national and international publications from 2000 to 2009 , of which 39 texts were identified to compose the study sample. The results showed a scarcity of publication on the theme, with the United States, Brazil, Canada, and the UK publishing most. The strategies found to attract blood donors were grouped as follows: welcoming; campaigns; and educational strategies. We observe the necessity for greater socialization of the strategies to attract blood donors utilized by hemocenters in order to exchange experiences between services and as such contribute to the increasing the number of blood donors in Brazil, seeking new donors and their loyalty.
\end{abstract}

DESCRIPTORS: Blood donors. Social marketing. Blood banks. Hemotherapy service.

\section{ESTRATEGIAS PARA RECLUTAR DONANTES DE SANGRE: UNA REVISIÓN BIBLIOGRÁFICA INTEGRADORA}

RESUMEN: Este estudio trata sobre una revisión bibliográfica integradora con el fin de conocer las estrategias que son utilizadas por los bancos de sangre para reclutar donantes. La revisión bibliográfica consideró las publicaciones nacionales e internacionales, de 2000 a 2009, donde se identificaron 39 textos que constituyeron la muestra del estudio. Los resultados mostraron la falta de publicaciones sobre el tema, y los países donde más se ha publicado son: los Estados Unidos, Brasil, Canadá y Reino Unido. Las estrategias desarrolladas para el reclutamiento de los donantes se agruparon como: estrategias de recepción, y, campañas y estrategias educativas. Se percibe la necesidad de una mayor socialización de las estrategias que son utilizadas por los bancos de sangre para reclutar los donantes, y de esa forma, contribuir a la captación de donantes de sangre, en la búsqueda de nuevos donantes y su lealtad.

DESCRIPTORES: Donadores de sangre. Mercadeo social. Bancos de sangre. Servicio de hemoterapia. 


\section{INTRODUÇÃO}

A doação de sangue é, ainda hoje, um problema de interesse mundial; pois não há uma substância que possa, em sua totalidade, substituir o tecido sanguíneo.

Os hemocentros têm dificuldades em manter o estoque de sangue para atender às necessidades específicas e emergenciais, colocando em risco a saúde e a vida da população.

As estatísticas mundiais mostram que as doações de sangue não acompanham o aumento de transfusões. Muitos países enfrentam dificuldades em suprir a demanda de sangue e hemocomponentes, principalmente, aqueles em que há uma política proibitiva em relação à comercialização do sangue, assim como o Brasil. ${ }^{1}$

O marketing é importante para a captação de doadores, sendo necessária a utilização de estratégias consistentes para tornar a doação de sangue, parte de hábitos e valores da população. A doação de sangue não faz parte da vida da maioria da população, por isso, é fundamental o planejamento, o desenvolvimento, a avaliação de estratégias e a sua socialização, possibilitando novas formas de captação, o que justifica o desenvolvimento dessa revisão integrativa. ${ }^{2}$ Com ela, pretende-se ampliar a visão sobre o tema, mesmo que o nosso maior interesse seja o contexto brasileiro.

Elegemos como questão norteadora para essa revisão: quais estratégias de captação de doadores de sangue são desenvolvidas pelos centros de hemoterapia? Dessa forma, nosso objetivo foi identificar o perfil das publicações relacionadas ao tema e conhecer as estratégias de captação de doadores de sangue desenvolvidas pelos hemocentros. Nesse contexto, esse estudo justifica-se por possibilitar a socialização de estratégias de captação de doadores de sangue, visando à contribuição dessas experiências para a busca de novos doadores e de sua fidelização.

\section{PROCEDIMENTOS METODOLÓGICOS}

Para o alcance dos objetivos desse estudo, optamos pelo método da revisão integrativa, uma vez que a mesma permite a busca, a avaliação crítica e a síntese das evidências disponíveis acerca do tema pesquisado, ${ }^{3}$ além de apontar lacunas do conhecimento, que poderão ser supridas com o desenvolvimento de novos estudos.

A revisão integrativa pode ser definida como aquela em que o autor da revisão está interessado em deduzir generalizações sobre determinadas questões, a partir de um conjunto de estudos, influenciando diretamente sobre elas. ${ }^{4}$

Utilizamos os seis passos propostos para a revisão integrativa: ${ }^{4}$ seleção das questões a serem estudadas, estabelecimento dos critérios para a seleção da amostra, representação das características do texto original, análise dos dados, interpretação dos resultados e apresentação da revisão.

Realizamos a busca por descritores nas bases de dados do editor EBSCO, que englobaram: CINAHL, CINAHL Plus with full text, Nursing and Allied Health, MEDLINE, MedicLatina, MEDLINE with full text, e da Biblioteca Virtual da SaúdeBIREME, que envolveu as bases LILACS, SciELO, BDENF, DESASTRES e MEDCARIB, utilizando como descritores: doadores de sangue, marketing social, bancos de sangue e/ou serviços de hemoterapia. Foi estabelecido o período de publicações, de janeiro de 2000 a dezembro de 2009. Resultaram dessa busca 14.687 estudos, sendo 8.092 na base de dados da EBSCO, e 6.595 estudos, na Biblioteca Virtual da Saúde-BIREME.

A revisão dos textos ocorreu em dois momentos, durante os meses de junho de 2008 a dezembro de 2009. No primeiro momento houve uma análise preliminar, tendo como critérios: estar em português, inglês ou espanhol e abordar a temática de captação de doadores. Esta seleção foi efetuada a partir da leitura do título dos artigos. Foram também excluídos os estudos duplicados e os estudos não disponíveis on-line, resultando em 3.617 textos, sendo 2.083 do Editor EBSCO, e 1.534 da Biblioteca Virtual da Saúde-BIREME.

A partir desta etapa, foi realizada a leitura dos resumos, a fim de identificar os estudos que atendiam aos seguintes critérios de inclusão: texto original (pesquisas e relatos de experiência), abordar estratégias de captação de doadores e o trabalho estar disponível na íntegra. Os critérios de exclusão foram: estudos clínicos ou estudos epidemiológicos. Neste processo de revisão participaram, além das duas autoras, seis pesquisadores voluntários, integrantes do grupo de pesquisa Educação em Enfermagem e Saúde (Eden), da Universidade Federal de Santa Catarina, que foram capacitados para tal atividade.

Deste processo, foram selecionados 39 artigos, que atenderam os critérios de inclusão e exclusão, para compor a tabela da Revisão Integrativa (37 da editora EBSCO e dois da BIREME). O principal item de exclusão foi a temática, pois a maioria dos artigos captados 
abordava aspectos técnicos sobre o sangue ou a doação de sangue, não focalizando as estratégias de captação de doadores.

As informações coletadas foram extraídas desta amostra, utilizando um quadro como instrumento, na qual sintetizamos as informações-chave. Nosso instrumento foi composto por número fornecido ao estudo, por ordem de localização; título do artigo; nome do periódico; ano de publicação; objetivo do estudo; referencial teórico, quando explicitado; estratégias adotadas para captação de doadores; tipo de estudo; resultados; e recomendações/conclusões, os quais foram preenchidos para cada artigo da amostra dessa revisão.

Para a análise dos dados utilizamos a sistematização das informações, estruturada a partir de dois momentos: no primeiro momento identificamos os dados relacionados ao perfil das publicações, sistematizando em uma tabela, contendo: título, autoria, ano de publicação, periódico, objetivo e metodologia. No segundo momento, o processo de análise se fundamentou em leitura interpretativa dos artigos, destacando as similaridades dos conteúdos e relacionando o que os autores apresentam sobre as estratégias utilizadas para a captação de doadores de sangue. A partir destas informações, focalizamos a compreensão dos seus resultados, com base na Política Nacional de Captação de Doadores de Sangue, ${ }^{5}$ a qual serviu como pano de fundo para a compreensão de outras realidades, além de nos permitir perceber pontos positivos e lacunas em relação à nossa própria realidade e política.

\section{RESULTADOS E DISCUSSÃO}

Constatamos, a partir das produções científicas, a dificuldade comum aos países em manter os estoques de sangue compatíveis com a demanda. Isso ocorre tanto por problemas de captação de doadores de sangue, o que implica no desenvolvimento de estratégias, como por problemas relacionados à contaminação por transfusão, colocando em risco a credibilidade da doação, dos hemocentros e do próprio sangue. Há três tipos de doadores nos países da América Latina; ${ }^{6}$ doadores de reposição, que representam a maioria, doadores remunerados, ainda reconhecidos em alguns países, e doadores voluntários, que representam uma pequena fração do total.

Os estudos revisados mostram que esses problemas têm ocorrido na China, na Venezuela e no Chile, ${ }^{7-8}$ onde, em 2000, as estatísticas mostra- ram que apenas $2,6 \%$ das doações, do total, foram provenientes de doações voluntárias ${ }^{9}$.

No Brasil, a política referente à hemoterapia proíbe a comercialização do sangue, o que ainda constitui um problema em alguns países, por colocar em risco o sangue coletado, em razão do interesse à remuneração pelo sangue doado, como na China, por exemplo. ${ }^{7}$ Apesar de aprovada em 1998, a lei nacional chinesa banindo a doação remunerada e encorajando a doação voluntária, ainda não foi aplicada, por dificuldades em colocar em prática a legislação, em razão da falta de uma cultura voltada à doação de sangue voluntária e altruísta.

A seguir, apresentamos a tabela 1 que se refere à distribuição de periódicos, conforme os estudos revisados.

Tabela 1 - Distribuição dos estudos revisados, por periódico. Florianópolis-SC, 2009

\begin{tabular}{lcc}
\hline Periódicos & $\mathbf{n}$ & \% \\
\hline Transfusion & 19 & 48,7 \\
Vox Sanguinis & 04 & 10,3 \\
Panamericana de Salud Publica & 03 & 7,6 \\
Transfusion Medicine & 03 & 7,6 \\
Texto \& Contexto Enfermagem & 02 & 5,1 \\
Cadernos de Saúde Pública & 02 & 5,1 \\
Canadian Medical Association Journal & 01 & 2,6 \\
Psycology, Health and Medicine & 01 & 2,6 \\
Eletrônica de Enfermagem & 01 & 2,6 \\
Journal of Advanced Nursing & 01 & 2,6 \\
BMC Public Health & 01 & 2,6 \\
Revista de la Facultad de Medicina & 01 & 2,6 \\
Universidad de Los Andes & & \\
\hline Total & 39 & $\mathbf{1 0 0}$ \\
\hline
\end{tabular}

Os três periódicos especializados na temática e que foram responsáveis por mais de 50 das publicações foram: o Transfusion, que é a principal publicação do mundo sobre novas informações a respeito da medicina transfusional; Vox Sanguinis, que é uma importante revista sobre transfusão sanguínea e transplante de outros tecidos; e a Transfusion Medicine que oferece informações científicas para hematologistas e hemoterapeutas, enfermeiros, técnicos e demais profissionais envolvidos no fornecimento de produtos derivados do sangue. 
Observamos que poucos periódicos brasileiros apresentaram artigos sobre estratégias de captação de doadores de sangue, sendo que a maioria aborda questões técnicas referentes ao sangue. Não encontramos qualquer periódico on-line específico sobre a temática, o que dificulta a troca de experiências entre os hemocentros. Há pouco estímulo à divulgação por parte das Políticas Nacionais de Doação Voluntária de Sangue.

Quanto às abordagens e tipos de estudos pesquisados, 13 são pesquisas quantitativas, 12 são qualitativas, dez são quali-quantitativas e quatro são relatos de experiência

O período investigado compreendeu 2000 a 2009 , sendo que na tabela 2 apresentamos a distribuição desses artigos, durante o período.

Tabela 2 - Distribuição temporal dos artigos analisados

\begin{tabular}{ccc}
\hline Período & $\mathbf{n}$ & $\%$ \\
\hline $2000-2003$ & 7 & $18 \%$ \\
$2004-2006$ & 11 & $28,2 \%$ \\
$2007-2009$ & 21 & $53,8 \%$ \\
\hline Total & $\mathbf{3 9}$ & $\mathbf{1 0 0}$ \\
\hline
\end{tabular}

O número de publicações tem aumentado gradativamente, mas ainda há a necessidade de mais publicações sobre o assunto. A divulgação de estratégias de captação de doadores de sangue poderá despertar nos leitores, profissionais da área, novas idéias e possibilidades, que possam contribuir para a captação e, dessa forma, aumentar o número de doadores.

Dentre as bases pesquisadas, o país que mais publicou sobre estratégias de captação de doadores de sangue foram os Estados Unidos, com 13 estudos, nos quais um deles aborda a realidade de 15 países da América Latina. Em seguida, os países que mais publicaram foram o Brasil, com seis estudos, o Reino Unido com quatro, e o Canadá, com três estudos. A Suécia publicou dois estudos e países como a Alemanha, a Austrália, o Chile, a China, a Espanha, a França, a Grécia, a Holanda, a Irlanda, a Lituânia e a Venezuela publicaram um estudo cada um.

A América foi o continente que mais apresentou publicações sobre estratégias de captação de doadores, compreendendo EUA, Chile, Canadá, Venezuela e Brasil, e totalizando 24 estudos. Em segundo lugar, temos o continente europeu, com
13 estudos, distribuídos entre Grécia, Espanha, Reino Unido, Alemanha, Suécia, França e Holanda. A Ásia e a Oceania, representadas pela China e Austrália, apresentaram um estudo cada um.

Quanto aos objetivos estabelecidos nos estudos revisados, verificamos que "compreender o que motiva o doador à doação e conhecer e avaliar as estratégias de captação de doadores" e "Discutir sobre as dificuldades da captação de doadores dos hemocentros, conhecer e avaliar a eficácia de estratégias/programas de captação de doadores" foram os principais objetivos desses estudos, conforme descritos na tabela 3. Este resultado nos permite reforçar, sobretudo, a importância da socialização de experiências e de pesquisas avaliativas sobre as estratégias de captação desenvolvidas para a busca de novos doadores de sangue e de sua fidelização.

Tabela 3 - Distribuição dos artigos científicos analisados conforme o objetivo central do estudo. Florianópolis-SC, 2009

\begin{tabular}{lcc}
\hline Objetivo & $\mathbf{n}$ & $\%$ \\
\hline $\begin{array}{l}\text { Compreender o que determina o retorno do } \\
\text { doador de sangue e conhecer suas motiva- }\end{array}$ & 14 & 35,9 \\
$\begin{array}{l}\text { Ções, atitudes, crenças e mitos. } \\
\begin{array}{l}\text { Discutir sobre as dificuldades da captação } \\
\text { de doadores dos hemocentros e conhecer e }\end{array}\end{array}$ & 12 & 30,8 \\
$\begin{array}{l}\text { avaliar a eficácia de estratégias/programas } \\
\text { de captação de doadores. }\end{array}$ & &
\end{tabular}

Apresentar experiências e sugestões para a captação de doadores de sangue, a fim de contribuirpara oaumento dos estoques desangue.

Compreender a visão de doadores e não doadores sobre os fatores que predispõem à baixa taxa de doação de sangue e ao impedimento à doação.

Argumentar sobre a importância das pesquisas para melhorar a captação de doadores de sangue.

Verificar as alterações na distribuição etária entre os doadores de sangue na população dos EUA.

Explorar as dificuldades e oportunidades que os hemocentros têm no recrutamento de jovens como doadores voluntários de sangue.

Explorar a perspectiva dos doadores de sangue sobre sua experiência de notificação para a não-doação, em função da inaptidão sorológica.

\begin{tabular}{lll}
\hline Total & 39 & 100 \\
\hline
\end{tabular}

Diversas foram as estratégias de captação pesquisadas, relatadas e sugeridas pelos autores, 
como a utilização de e-mail, ${ }^{10-11}$ através do qual possibilitou, também, o contato do doador com o hemocentro, para agendamento de doação, ${ }^{12}$ programa de marketing por telefone, ${ }^{7-8,10}$ e o oferecimento de presentes e benefícios, como, camisetas, ${ }^{14}$ canecas, pins, certificados, adesivos, além de exames médicos, como o colesterol, ${ }^{15}$ foram outras estratégias utilizadas. A solicitação pessoal, por telefone ou por e-mail, foi constatada como uma estratégia positiva para a captação de doadores. ${ }^{12,16}$

Segundo uma pesquisa realizada na Lituânia, ${ }^{17}$ o comércio de sangue contribui para o aumento significativo das doações e, sem ele, a maioria dos doadores investigados doaria, em caso de emergência, para familiares e/ou amigos, ou ainda não doaria mais sangue. Não é o caso do Brasil, onde o comércio de sangue é proibido e há a preocupação com a educação e conscientização sobre a importância da doação voluntária de sangue junto à população, especialmente à população jovem. ${ }^{18}$ Da mesma forma, em resultados de uma pesquisa desenvolvida nos EUA, ${ }^{12}$ foi percebido que o benefício monetário prejudica a doação futura, no sentido de impactar negativamente, como estratégia de captação de doadores de sangue.

\section{Estratégias de captação de doadores}

A análise crítica dos resultados obtidos, a partir dos estudos revisados, apontou três categorias de estratégias de captação de doadores de sangue, que são: acolhimento, campanhas e estratégias educativas.

O acolhimento: o bom atendimento aos doadores foi apontado como possibilidade de fidelizá-los, ${ }^{19}$ além da ampliação de coletas, facilitando o retorno do doador. ${ }^{20}$ Remover barreiras que dificultam a doação, assim como aumentar a oportunidade de coletas, especialmente em locais de trabalho, com a devida dispensa, são fatores que contribuem para a conquista e a retenção de doadores. ${ }^{21}$ Uma pesquisa apontou que o fácil acesso à doação, em locais como igrejas e supermercados, foi um dos motivos que mais mobilizou os doadores à doação de sangue. ${ }^{22} \mathrm{~A}$ mesma pesquisa apontou como fator desmotivador o medo da agulha, o que pode ser minimizado com a capacitação de técnicos para a punção da veia. Sensibilizar o doador a incentivar um amigo ou familiar para a primeira doação foi considerada como uma boa estratégia de captação. ${ }^{23}$

As campanhas são estratégias pontuais que ocorrem por um determinado tempo e por alguma razão específica. Geralmente, apresentam resulta- dos satisfatórios, como a promovida em Berlin, ${ }^{24}$ com a participação do último descendente do Conde Drácula, para autógrafos, após sessão do filme, apresentando bons resultados durante e logo após a sua execução.

No Reino Unido, campanhas nacionais de televisão e rádio focaram em celebridades, as quais contaram como elas ou um de seus parentes foram ajudados com a doação de sangue; enfatizando frases como: "faça algo incrível, doe sangue." ${ }^{25}$ Campanhas realizadas com o auxílio da mídia também socializaram informações sobre a doação. ${ }^{26-27}$

Algumas publicações sugerem estudos de mercado social através de uma estratégia para detectar atitudes em relação à doação de sangue e guiar ações para a captação de doadores voluntários, ${ }^{8,28-33}$ assim como pesquisas que investigam o que impede a doação de sangue. ${ }^{34}$ Estudos mercadológicos na área da hemoterapia brasileira visam a contribuir com o Programa Nacional de Doação de Sangue (PNDVS) ${ }^{35}$ e sensibilizar as instituições para aprimorar a captação de doadores e tornar o doador "de repetição".

Outro exemplo de campanha educativa foi a baseada no desenho animado do "Super-herói Defensor Vermelho", que não pode salvar todas as vidas e necessita da ajuda dos doadores. Campanhas semelhantes a essa são motivadas pela força do altruísmo, ${ }^{36}$ em que se combinam o senso de recompensa pessoal e o desejo de ajudar. Estudos mostram que as pessoas expostas às mensagens altruístas estão mais propensas a doar, no futuro.

Estratégias educativas são mais efetivas. Vídeos educativos sobre a necessidade da doação de sangue foi uma das estratégias apontadas nos EUA, tais como os enviados para a população americana, com descendência africana, onde há muitos casos de crianças com doença falciforme. ${ }^{37}$ Após seis meses, houve um incremento de 75\% no número total de doações; que é comparado ao número de doações do mesmo período do ano anterior, em que existiram $64 \%$ de acréscimo no número da primeira doação da população para a qual foi enviada o vídeo educativo.

Outra estratégia sugerida foi a educação direcionada com conhecimentos científicos, podendo encorajar futuros doadores, ${ }^{7}$ devendo ter início na formação de hábitos do jovem, enfocando a doação como um ato responsável, de maturidade e de participação social. ${ }^{38}$

Há estudos que apontam a necessidade de conhecer e de utilizar teorias de psicologia, sociologia e antropologia, a fim de contribuírem 
para a compreensão de crenças, valores e atitudes do doador. ${ }^{12,34,39-40}$

Alguns autores sugerem estratégias à população, aos profissionais de saúde e aos bancos de sangue, como parcerias com os diversos setores da sociedade, capacitação dos profissionais da saúde que atuam junto ao doador e à educação à distância aos trabalhadores; objetivando a motivação ao trabalho. ${ }^{41}$ Sugerem, ainda, o desenvolvimento de programas com crianças, objetivando que elas sejam educadas e sensibilizadas à doação, no futuro. À exemplo dessa sugestão, um dos estudos revisados ${ }^{18}$ relata a experiência do Projeto Escola, do Centro de Hematologia e Hemoterapia de Santa Catarina, sendo uma estratégia de política pública, desenvolvida junto à população escolar, contribuindo, positivamente, para a transformação da cultura sobre a doação de sangue.

Uma pesquisa realizada nos EUA ${ }^{42}$ aponta para a importância de melhorar a captação e fidelização de jovens e adultos, em razão do envelhecimento da população doadora e da diminuição no índice de doação entre doadores mais jovens.

\section{CONSIDERAÇÕES FINAIS}

A maioria dos estudos revisados constituiuse de pesquisas, a fim de conhecer o comportamento dos doadores em relação à doação de sangue, ${ }^{7,9-11,20,25,29-30,41-45}$ dos que deixaram de doar e daqueles que nunca doaram. A pesquisa é uma estratégia fundamental para a obtenção de subsídios para o direcionamento de ações para a captação de doadores de sangue, pois, conhecer suas concepções, valores e sentimentos é essencial para melhorar a captação e fidelização do doador. Ao conhecer o doador, há a facilitação ao atendimento de suas necessidades, contribui para a sua satisfação e, consequentemente, para o aumento da população doadora de sangue. A informação de teorias de psicologia, sociologia e antropologia poderá auxiliar esse processo.

Os estudos apontam como estratégia em potencial, com resultados imediatos, o uso da mídia pessoal, através do marketing, por meio de e-mails e de telefonemas; possibilitando novos registros de doação, além de ser uma ferramenta de marketing organizacional de custo baixo, porém, é uma ferramenta pontual. $\mathrm{O}$ bom atendimento ao doador é uma estratégia eficaz e necessita da capacitação e da boa vontade dos profissionais. Oferecer melhores condições ao doador, como a ampliação dos horários de atendimento, maior disponibilidade de coletas externas e de funcionários, faz parte da boa acolhida ao doador de sangue. ${ }^{45}$

As campanhas são estratégias que contribuem para a conquista de doadores, porém, apresentam efeitos positivos logo após a sua realização.

Ressaltamos que estratégias educativas proporcionam resultados mais efetivos para a doação de sangue, podendo apresentar efeitos à curto, médio e longo prazos.

Brindes e benefícios ao doador não devem constituir estratégias para conquistar doadores, pois a doação de sangue deve ser um ato sem interesse por algo em troca.

Ressaltamos a importância sobre a questão do envelhecimento da população, visto que este é um fator que nos leva à necessidade de desenvolver estratégias educativas junto aos mais jovens; a fim de sensibilizá-los para a necessidade da doação de sangue e, dessa forma, buscarmos garantir a segurança transfusional.

Muitos países precisam evoluir em relação à política da doação voluntária de sangue, pois diversos problemas relacionados à doação e à transfusão sanguínea necessitam de maior atenção, para possibilitar maior segurança à população.

Sendo assim, ressaltamos a importância das autoridades para o incentivo ao desenvolvimento de projetos que visem à educação da população para a doação de sangue, especialmente, os que desenvolvem seus trabalhos junto aos jovens, pois estes serão os nossos futuros doadores. Além de atenderem aos preceitos das Políticas do Sangue e Hemoderivados, ${ }^{5}$ contribuirão para a formação do cidadão consciente de seu papel na sociedade.

\section{REFERÊNCIAS}

1. Brasil. Constituição da República Federativa do Brasil de 1988, de 5 de outubro de 1988. [online]. [acesso 2009 Dez 15]. Disponível em: http:// www.planalto.gov.br/ccivil_03/constituicao/ constitui\%C3\%A7ao.htm

2. Mendes KDS, Silveira RCCP, Galvão CM. Revisão integrativa: método de pesquisa para a incorporação de evidências na saúde e na enfermagem. Texto Contexto Enferm. 2008 Out-Dez; 17(4):758-64.

3. Jackson GB. Methods for integrative reviews. Rev Educ Res. 1980 Fall; 50(3):438-60.

4. Ganong LH. Integrative reviews of nursing research. Res Nurs Heath. 1987 Fev; 10(1):1-11.

5. Agência Nacional de Vigilância Sanitária. Política Nacional de Sangue e Hemoderivados do Ministério 
da Saúde [online]. [acesso 2009 Dez 15]. Disponível em http://www.anvisa.gov.br/divulga/noticias.htm

6. Gutiérrez MG, Tejada ES, Cruz JR. A study of sociocultural factors related to voluntary blood donation in the Americas. Rev Panamer Salud Pública. 2003 Feb-Mar; 13(2/3):85-90.

7. Tison GH, Liu C, Ren F, Nelson K, Shan H. Influences of general and traditional Chinese beliefs on the decision to donate blood among employerorganized and volunteer donors in Beijing, China. Transfusion. 2007 Oct; 47(10):1871-9.

8. De Oliveira MCA. Experiencias en el Programa de Sangre de la Cruz Roja en Venezuela. MedULA. 2008 Jan; 17(1):58-62.

9. Vásquez M, Ibarra P, Maldonado M. Conocimientos y actitudes hacia la donación de sangre en una población universitaria de Chile. Rev Panam Salud Públ. 2007 Nov; 22(5):323-8.

10. Glynn SA, Schreiber GB, Murphy EL, Kessler D, Higgins $M$, Wright DJ, et al. Factors influencing the decision to donate: racial and ethnic comparisons. Transfusion. 2006 Jun; 46(6):980-90.

11. Marantidou O, Loukopoulou L, Zervou E, Martinis G, Egglezou A, Fountouli P, et al. Factors that motivate and hinder blood donation in Greece. Transfus Med. 2007 Dec; 17(6):443-50.

12. Rapport FL, Maggs CJ. Titmuss and the gift relationship: altruism revisited. J Adv Nurs. 2002; 40(5):495-503.

13. Gonçalez T, Sabino EC, Chamone DF. Trends in the profile of blood donors at a large blood center in the city of São Paulo, Brazil. Rev Panamer Salud Pública. 2003 Feb-Mar; 13(2/3):144-8.

14. Reich P, Roberts P, Laabs N, Chinn A, McEvoy $\mathrm{P}$, Hirschler N, et al. A randomized trial of blood donor recruitment strategies. Transfusion. $2006 \mathrm{Jul}$; 46(7):1090-6.

15. Glynn SA, Williams AE, Nass CC, Bethel J, Kessler $\mathrm{D}$, Scott EP, et al. Attitudes toward blood donation incentives in the United States: implications for donor recruitment. Transfusion. 2003 Jan; 43(1):7-16.

16. Simon TL. Where have all the donors gone? A personal reflection on the crisis in America's volunteer blood program. Transfusion. 2003 Feb; 43(2):273-9.

17. Buciuniene I, Stonienë L, Blazeviciene A, Kazlauskaite $\mathrm{R}$, Skudiene $\mathrm{V}$. Blood donors' motivation and attitude to non-remunerated blood donation in Lithuania. BMC Public Health. 2006 Jun; 6:166.

18. Pereima RSM, Arruda MW, Reibnitz KS, Gelbcke FL. Santa Catarina Hemotological and Hemotherapy Center School Project: a public policy strategy. Texto Contexto Enferm. 2007 Jul-Sep; 16(3):546-52.

19. Nguyen DD, Devita DA, Hirschler NV, Murphy EL. Blood donor satisfaction and intention of future donation. Transfusion. 2008 Apr; 48(4):742-8.
20. Schlumpf KS, Glynn SA, Schreiber GB, Wright DJ, Randolph Steele W, Tu Y, et al. Factors influencing donor return. Transfusion. 2008 Feb;48(2):264-72.

21. McVittie C, Harris L, Tiliopoulos N. "I intend to donate but ...": non-donors' views of blood donation in the UK. Psychol Health Med. 2006 Feb; 11(1):1-6.

22. Misje AH, Bosnes V, Heier HE. Recruiting and retaining young people as voluntary blood donors. Vox Sang. 2008 Feb; 94(2):119-24.

23. Davey RJ. Recruiting blood donors: challenges and opportunities. Transfusion. 2004 Apr; 44(4):597-600.

24. Kezwer G. Dracula wants your blood. Now!. Can Med Assoc J. 2000 Jan; 162(2):246-246.

25. Suárez IMB, Fernández-Montoya A, Fernández AR, López-Berrio A, Cillero-Peñuela M. How regular blood donors explain their behavior. Transfusion. 2004 Oct; 44(10):1441-6.

26. Mathew SM, King MR, Glynn SA, Dietz SK, Caswell SL, Schreiber GB. Opinions about donating blood among those who never gave and those who stopped: a focus group assessment. Transfusion. 2007 Apr; 47(4):729-35.

27. Moog R, Fourné K. Recruitment of prospective donors: what do they expect from a homepage of a blood transfusion service? Transfus Med. 2007 Aug; 17(4):279-84.

28. Nilsson Sojka B, Sojka P. The blood-donation experience: perceived physical, psychological and social impact of blood donation on the donor. Vox Sang. 2003 Feb; 84 (2):120-8.

29. Steele WR, Schreiber GB, Guiltinan A, Nass C, Glynn SA, Wright DJ, et al. The role of altruistic behavior, empathetic concern, and social responsibility motivation in blood donation behavior. Transfusion. 2008 Jan; 48(1):43-54.

30. Whittaker S, Carter N, Arnold E, Shehata N, Webert $\mathrm{KE}$, et al. Understanding the meaning of permanent deferral for blood donors. Transfusion. 2008 Jan; 48(1):64-72.

31. Benetti SRD, Lenardt MH. Meaning attributed to the blood for donors and receivers of blood. Texto Contexto Enferm. 2006 Jan-Mar; 15(1):43-50.

32. Cunha BGF, Dias MR. Comunicações persuasivas e doação regular de sangue: um estudo experimental. Cad. Saúde Pública. 2008 Jun; 24(6):1407-18.

33. Ludwig ST, Rodrigues ACM. Doação de sangue: uma visão de marketing. Cad. Saúde Pública. 2005 Mai- Jun; 21(3):932-9.

34. Harrington M, Sweeney MR, Bailie K, Morris K, Kennedy A, et al. What would encourage blood donation in Ireland? Vox Sang. 2007 May; 92(4): 361-7.

35. Ministério da Saúde (BR). Coordenação de Sangue e Hemoderivados. Meta Mobilizadora Nacional: sangue - $100 \%$ com garantia de qualidade em todo 
o seu processo até 2003. Brasília (DF): Programa Nacional de Doação Voluntária de Sangue; 1998b.

36. Ferguson E, France CR, Abraham C, Ditto B, Sheeran P. Improving blood donor recruitment and retention: integrating theoretical advances from social and behavioral science research agendas. Transfusion. 2007 Nov; 47(11):1999-2010

37. Price CL, Boyd JH, Watkins AR, Fleming F, DeBaun MR. Mailing of a sickle cell disease educational packet increases blood donors within an African American community. Transfusion. 2006 Aug; 46(8):1388-93.

38. Dos Santos NLP, Stipp MAC, Leite JL, Nunes AS. The elderly blood donor and the hemotherapic nurse's care. Rev Eletr Enferm [online]. 2008 [acesso 2009 Dez 15]. 10(3):828-34. Disponível em: http:// www.fen.ufg.br/revista/v10/n3/v10n3a29.htm.

39. Lemmens $\mathrm{KPH}$, Abraham C, Hoekstra T, Ruiter RAC, De Kort WLA, Brug J, et al. Why don't young people volunteer to give blood? An investigation of the correlates of donation intentions among young nondonors. Transfusion. 2005 Jun; 45(6):945-55.
40. Masser BM, White KM, Hyde MK, Terry DJ. The psychology of blood donation: current research and future directions. Transf Med Rev. 2008 Jul; 22(3):215-33.

41. Godin G, Conner M, Sheeran P, Bélanger-Gravel A, Germain M. Determinants of repeated blood donation among new and experienced blood donors. Transfusion. 2007 Sep; 47(9):1607-15.

42. Zou S, Musavi F, Notari EP, Fang CT. Changing age distribution of the blood donor population in the United States. Transfusion. 2008 Feb; 48(2):251-7.

43. Geyer ME. The impact of e-mail in acquiring and retaining whole-blood donors: a comparative analysis of the puget sound blood center donor e-mail communication program. Transfusion. 2005 Dec; 45(12):1957-64.

44. Danic B, LefrèreJJ. Transfusion and blood donation on the screen. Transfusion 2008 May; 48(5):1027-31.

45. Sojka BN, Sojka P. The blood donation experience: self-reported motives and obstacles for donating blood. Vox Sang. 2008 Jan; 94(1):56-63.

E-mail: rosanesuely@gmail.com 